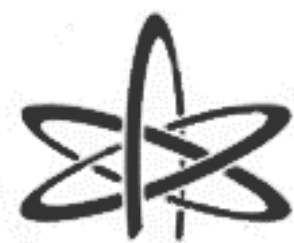

BJRS
BRAZILIAN JOURNAL

$\mathrm{OF}$

RADIATION SCIENCES

08-03A (2020) 01-18

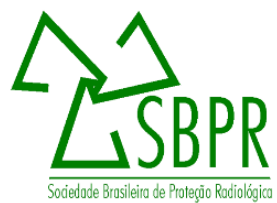

\title{
Dynamic mode decomposition of numerical data in natural circulation
}

\author{
$\operatorname{Ramos}^{\mathrm{a}}$ E. M. , Nascimento ${ }^{\mathrm{b}}$ F. R. T. , $\operatorname{Darze}^{\mathrm{b}}$ G. M. \\ Faccini $^{\mathrm{b}}$ J. L. H. , Giraldi ${ }^{\mathrm{a}}$ G. A. \\ ${ }^{a}$ National Laboratory for Scientific Computing - LNCC, 25651-075, Petrópolis, Rio de Janeiro, Brazil \\ Nuclear Engineering Institute - IEN/CNEN, 21941-906, Rio de Janeiro, Brazil \\ eliaquimgauss@hotmail.com
}

\begin{abstract}
Dynamic mode decomposition (DMD) has been used for experimental and numerical data analysis in fluid dynamics. Despite of its advantages, the application of the DMD methodology to investigate the natural circulation in nuclear reactors is very scarce in literature. In this paper it is applied the traditional DMD and its variation, the sparsity-promoting dynamic mode decomposition (SPDMD), for analysis of temperature and velocity fields. These datasets are generated by computational simulation of an experimental setup in reduced scale, similar to a heat removal system by natural circulation of a pool-type research reactor. Firstly the numerical data is partitioned, using a space-time correlation approach, in order to identify fundamental sequences to compute the dynamic modes. Next, the DMD and SPDMD methodologies are applied over each subsequence to obtain the dynamic modes of the temperature and velocity fields. Finally the flow fields are reconstructed and compared with the original numerical data. The conclusion is that the SPDMD performs better than DMD to represent both the temperature and velocity data.
\end{abstract}

Keywords: DMD, natural circulation, numerical data. 


\section{INTRODUCTION}

The natural circulation is a very important matter with great interest in the nuclear reactor thermal-hydraulics. Many studies concerning the heat removal of a nuclear reactor by natural circulation have been carried out in the last two decades, to more clearly understand the physical phenomena and to develop methods for simulating the thermal hydraulic behavior in a passive reactor cooling mode [1-4]. Theoretical models for prediction of single-phase and two-phase natural circulation flow parameters have been developed. Although these models have the ability to predict important flow parameters such as the pressure gradient, average phase velocities and void fractions, they are not capable to predict the flow structure itself. Moreover, the flow structure evolution may differ from that of forced convection flows [5], [6]. When a nuclear reactor is submitted to natural circulation conditions, a movement of the working fluid occurs from the hottest regions to the colder ones, resulting in a heat removal from the hottest regions. When this phenomenon is established, it has a heat exchange cycle that does not depend on any external mechanisms, for example, a pump. From this moment, the velocity fields control depends only on the phenomenon and any variation in the velocities should be attributed to buoyancy forces, which can be related with temperature variations in the system. In a particular case of a pool type research reactor after a shutdown, the heat is transferred by natural circulation from the core to a pool water upward through the core [7].

More recently the occurrence of the natural circulation in nuclear research reactors has been described in the literature by studies focusing mainly: the prevention of a severe accident [8], the effect of an unprotected reactivity insertion on the dynamic response of Materials Testing Reactor MTR research reactors under natural circulation regime [9], the investigation of flow reversal from downward forced to upward natural circulation [10], the stability of the natural circulation and its suppression due to the presence of oscillations [11], [12].

Since its proposal in [13], the dynamic mode decomposition (DMD) has been used for numerical and experimental data analysis in fluid mechanics to identify low-order dynamics. The DMD formulates the flow time series as a Krylov sequence [14] by assuming a linear mapping that connects the flow field at a time step $t$ to the subsequent flow field at time $t+\Delta t$. Then, given a 
sequence of $N$ flow snapshots, the DMD technique performs the singular value decomposition (SVD) of the data matrix composed by the first $N-1$ flow snapshots and applies preprocessing step using the SVD result to get a robust computation of the proper modes of the data sequence. The DMD approach and its variant, named sparsity-promoting DMD (SPDMD) [15], have been successfully applied for numerical data generated through Navier-Stokes codes and experimental data measurements.

In the standard DMD as well as in the SPDMD the reasoning to set $N$ is that, adding further flow fields $\mathbf{v}_{i}$ to the data sequence will not improve the vector space spanned by $V_{1}^{N}$. However, if we consider a transient regime we may have a data sub-sequence (with $N_{T}$ time steps) capturing the transient dynamics and a second sequence (with $N_{S}$ time steps) representing the quasi-stationary flow. Depending on the pattern complexity inside each flow regime the space spanned by one may (or may not) contains the other one as a subspace. This issue is particularly interesting to be investigated in natural circulation flows due to the possibility to assess itself the flow structure to study instabilities (oscillations), in single-phase and two-phase flows, as they can cause damages to the system due excessive pressure, temperature or vibration variation.

In this paper, as case study, we apply the DMD and SPDMD methodologies on numerical data, composed by time-varying temperature and velocity, generated by computational simulation of an experimental setup working under single-phase natural circulation flow. So, we apply the methodology proposed in [19] in order to set the best value for $N$. Next, we take each flow field and compare DMD and SPDMD regarding to flow patterns preservation and dimension of dynamic mode representation (compact representation). In the case of temperature we notice that both SPDMD and DMD reconstructed data preserve the flow fields but the former allows a more sparse (compact) representation. The same was verified for the velocity field. Consequently, we conclude that SPDMD outperforms traditional DMD in the performed tests. In the text remaining, we firstly summarize the DMD and SPDMD in section 2. Then, section 3 describes the methodology proposed in [19]. The computational setup is discussed in section 4. The results are presented in section 5. Finally, we show conclusions and future works (section 6). 


\section{DMD AND SPDMD APPROACHES}

In the DMD theory [13], a flow field in time step $i$ (numerical frame), is arranged in vector form $\mathbf{v}_{i} \in \mathbb{R}^{n}$. Then, we assemble the first $N$ snapshots in the form $V_{1}^{N}=\left\{\mathbf{v}_{1}, \mathbf{v}_{2}, \cdots, \mathbf{v}_{N}\right\} \in \mathbb{R}^{n \times N}$. If we suppose that $V_{1}^{N-1}$ is a rank - $r$ matrix, we can compute the economy-size singular value decomposition SVD of $V_{1}^{N-1}$ to get $V_{1}^{N-1}=U \Sigma W^{H}$ where $U \in \mathbb{R}^{n \times r}, \Sigma \in \mathbb{R}^{r \times r}, W \in \mathbb{R}^{(N-1) \times r}$, and $W^{H}$ denotes the transpose (or complex - conjugate transpose in $\mathbb{C}$ ).

Now, following [15], we define $F_{d m d}=U^{H} V_{2}^{N} W \Sigma^{-1} \in \mathbb{R}^{r x r}$ and solve the eigenvectoreigenvalue equation $F_{d m d} \mathbf{y}=\mu \mathbf{y}$. Then, the dynamic mode $\phi \in \mathbb{C}^{n}$ is defined by [13], [15]:

$$
\phi=U \mathbf{y} .
$$

If we suppose that $F_{d m d} \in \mathbb{R}^{r \times r}$ has a full set of linearly independent eigenvectors $Y=$ $\left\{\mathbf{y}_{1}, \mathbf{y}_{2}, \cdots \mathbf{y}_{r}\right\}$ with corresponding eigenvalues $\left\{\mu_{1}, \mu_{2}, \cdots, \mu_{r}\right\}$, we can write $\mathbf{v}_{t+1} \approx \sum_{i=1}^{r} \zeta_{i}^{t} \phi_{i}, t=0,1,2, \ldots, N-2$,[15]. If we write $\zeta_{i}^{t}=\mu_{i}^{t} \alpha_{i}, i=1,2, \cdots, r$, then, we must determine the optimum vector of amplitudes $\alpha=\left[\alpha_{1} \alpha_{2} \cdots \alpha_{r}\right]^{T}$ to represent the time sequence $V_{1}^{N-1}$. The optimum $\alpha$ can be found by solving the problem:

$$
\alpha_{d m d}=\arg \min _{\alpha}\left\|V_{1}^{N-1}-\Phi D_{\alpha} V_{a n d}\right\|_{F}^{2}
$$

where $\Phi=\left[\begin{array}{lllll}\phi_{1} & \phi_{2} & \phi_{3} & \cdots & \phi_{r}\end{array}\right]=U Y, D_{\alpha}=\operatorname{diag}\left(\alpha_{1}, \alpha_{2}, \cdots, \alpha_{r}\right)$, and $V_{\text {and }}$ is the Vandermonde matrix given by $V_{\text {and }}(i, j)=\mu_{i+1}^{j}, 0 \leq j \leq N-2$. In [15] it is demonstrate that the solution of problem (2) is:

$$
\alpha_{d m d}=\left[\left(Y^{H} Y\right) \circ\left(\overline{V_{a n d} V_{a n d}^{H}}\right)\right]^{-1} \overline{\operatorname{diag}\left(V_{a n d} W \Sigma^{H} Y\right)},
$$

where the operation ' $\circ$ ' means the elementwise multiplication of two matrices. 
The key idea behind SPDMD is to change the objective function in expression (2) in order to introduce sparsity in the dynamic mode representation but keeping the quality of the reconstruction. This is implemented by solving the optimization problem:

$$
\widehat{\alpha}_{s p d m d}=\arg \min _{\widehat{\alpha}}\left(\left\|V_{1}^{N-1}-\Phi \widehat{D}_{\alpha} V_{a n d}\right\|_{F}^{2}+\gamma \sum_{i=1}^{r}\left|\widehat{\alpha}_{i}\right|\right),
$$

where $\widehat{D}_{\alpha}=\operatorname{diag}\left(\hat{\alpha}_{1}, \hat{\alpha}_{2}, \cdots \hat{\alpha}_{r}\right), \Phi$ and $V_{\text {and }}$ are the same matrices of expression (2), and $\gamma \in \mathbb{R}$ controls the sparsity of the solution. Once problem (4) is solved, the second step of SPDMD technique adjusts the values of the non-zero entries of $\hat{\alpha}$ in order to optimally approximate the entire data sequence. That means, we solve the problem (4) with $\gamma=0$, but subject to $E^{T} \hat{\alpha}=0$ where $E \in \mathbb{R}^{r \times m}$ encodes information about the sparsity structure of the vector $\hat{\alpha}$ (see [15] for details).

\section{METHODOLOGY}

A critical value in the DMD techniques of previous section is the sub-sequence size $N$. So, in order to compute $N$, we apply the method presented in [19] and firstly perform a coarse temporal data segmentation using a simple similarity measure, the cross-correlation defined by [16]:

$$
\begin{aligned}
C_{i, i+1} & =\frac{\left(\mathbf{v}_{i+1}-\bar{v}_{i+1} \mathbf{e}\right) \cdot\left(\mathbf{v}_{i}-\bar{v}_{i} \mathbf{e}\right)}{\left\|\mathbf{v}_{i+1}-\bar{v}_{i+1} \mathbf{e}\right\|\left\|\mathbf{v}_{i}-\bar{v}_{i} \mathbf{e}\right\|}, \\
\text { where } \bar{v}_{i}=\left(\sum_{j=1}^{n} v_{i, j}\right) / n \text { and } \mathbf{e} & =\left[\begin{array}{llll}
1 & 1 & \ldots & 1
\end{array}\right]^{T}
\end{aligned}
$$

The lower/higher values of the cross-correlation (5) indicate the evolution in time of different flow configurations, given a guess about fundamental sub-sequences. To smooth the correlation signal, we apply the total variation filter [20], generating a smooth signal $T(C)_{i, i+1}$. This iterative 
technique depends on two parameters: smoothing parameter $\lambda$ and number of iterations $N_{i t}$, which we set by trial and error.

The obtained function is the input for a discrete differentiation filter, combined with a simple thresholding operation, that highlights the points of transition in the numerical frame sequence. Let us suppose that such transitions happen for $k \in\left\{i_{1}, i_{2}, \cdots, i_{M}\right\}$, with $i_{1}<i_{2}<\cdots<i_{M}$. From this result, we generate $M$ segments given by the subsequences $\left[1, i_{1}\right],\left[1, i_{2}\right], \cdots,\left[1, i_{M}\right]$. We take into account the rank of each segment obtained in order to compute the DMD and SPDMD dynamic modes for each interval $[1, k]$, denoted by $\phi_{i, k}$ and $\hat{\phi}_{i, k}$, respectively. Then, each segment is analyzed considering the norm of the residuals of DMD, computed by the frame-to-frame error $\left(E_{d m d}\right)$ and the root mean square error $\left(R M S E_{d m d}\right)$ given, respectively, by:

$$
\begin{gathered}
E_{d m d}(k, t)=\left\|\frac{\mathbf{v}_{t+1}-\sum_{i=1}^{n} \phi_{i, k} \mu_{i, k}^{t} \alpha_{i, k}}{H^{2}}\right\|, \\
R M S E_{d m d}(k, \theta)=\sqrt{\frac{\sum_{t=0}^{\theta-2} E_{d m d}(k, t)^{2}}{\theta-1}}
\end{gathered}
$$

where $H^{2}$ is the dimension of the data vectors, $k \in\left\{i_{1}, i_{2}, \cdots, i_{M}\right\}$, and $\theta \in\left\{i_{1}, i_{2}, \cdots, i_{M}, N\right\}$. When $\theta \neq N$ we call the result a local reconstruction error, otherwise $(\theta=N)$ we say that we calculate global reconstruction errors. The idea is to choose an interval with low global and low local reconstruction errors. We can choose the best $\left[1, i_{\text {opt }}\right]$ interval to compute the DMD dynamic modes by solving the optimization problem [19]:

$$
i_{\text {opt }}=\arg \min _{k \in\left\{i_{1}, i_{2}, \ldots, i_{M}\right\}} \mathcal{L}(k, N)
$$

where:

$$
\mathcal{L}(k, N)=R M S E_{d m d}(k, N)+R M S E_{d m d}(k, k),
$$


since, the first term $R M S E_{d m d}(k, N)$ is the global reconstruction error obtained when computing DMD using the interval $[1, k]$ and the second term $R M S E_{d m d}(k, k)$ is the local reconstruction error in the same interval.

However, to compute $R M S E_{d m d}(k, N)$ we need to calculate DMD technique using the interval $[1, k]$ but reconstruct $(N-1)>k$ frames using the obtained dynamic modes. To perform this task, we must solve the optimization problem (2) but, in this case, $\Phi$ is related to the SVD decomposition of $V_{1}^{k-1}$ instead of $V_{1}^{N-1}$. We solve this generalized version of problem (2) by noticing that (see [19]):

$$
\mathbf{v}_{t+1} \approx\left[\mu_{1}^{t} \phi_{1}, \quad \mu_{2}^{t} \phi_{2}, \ldots, \quad \mu_{r}^{t} \phi_{r}\right] \alpha
$$

Hence, if we define $\Psi^{(t)} \equiv\left[\begin{array}{llll}\mu_{1}^{t} \phi_{1} \mu_{2}^{t} \phi_{2} & \cdots & \mu_{r}^{t} \phi_{r}\end{array}\right]$, we can follow [19] and compute the solution of this generalized version of problem (2) through:

$$
\alpha_{o p t}=B^{-1} \mathbf{c}^{T}
$$

where $B=\sum_{t=1}^{N-1}\left[\Psi^{(t-1)}\right]^{T} \Psi^{(t-1)}$ and $\mathbf{c}=\sum_{t=1}^{N-1} \mathbf{v}_{t}^{T} \Psi^{(t-1)}$

Considering that the SPDMD tries to optimize the data representation in the dynamic mode subspace, we take the solution of (8) to compute the SPDMD technique. We follow the SPDMD methodology available in [18] and seek for a near-optimum value for parameter $\gamma$ by considering a log-distributed list of values. Then, we take each dynamic mode subspace generated and compare the global reconstruction error as well as the sparsity of the vector $\alpha$ versus the $\gamma$ values. The SPDMD frame-to-frame error $E_{s p d m d}$ and the corresponding root mean square error $R M S E_{s p d m d}$ have the same form of expressions (6)-(7):

$$
E_{s p d m d}(k, t)=\left\|\frac{\mathbf{v}_{t+1}-\sum_{i=1}^{r} \widehat{\phi}_{i, k} \widehat{\mu}_{i, k}^{t} \widehat{\alpha}_{i, k}}{H^{2}}\right\|,
$$




$$
R M S E_{\text {spdmd }}(k, \theta)=\sqrt{\frac{\sum_{t=0}^{\theta-2} E_{\text {spdmd }}(k, t)^{2}}{\theta-1}},
$$

and, like for DMD, the global reconstruction error associated to the optimum interval $\left[1, i_{\text {opt }}\right]$ is computed by $R M S E_{d m d}\left(i_{o p t}, N\right)$. Likewise in the DMD case, we must compute expression (10), with the vector $\mathbf{c}$ and matrix $B$ yielded using the SPDMD dynamic modes with $\hat{\alpha}_{i} \neq 0$. In this case, we want to obtain a desirable tradeoff between the quality of approximation and the number of modes that are used to approximate the time series. Therefore, we set $\gamma$ with a value that gives low global reconstruction error as well as high sparsity.

Next, we take each data sequence and compare DMD and SPDMD regarding to the flow pattern preservation and compact representation. Both techniques are computed using the best interval $\left[1, i_{\text {opt }}\right]$.

\section{EXPERIMENTAL AND COMPUTATIONAL SETUPS}

The experimental setup is shown in Figure 1. It was designed according to similarity criteria for scaling reduced models in relation to a prototype of a pool type $30 \mathrm{MW}$ research reactor. It consists of a cylindrical vessel with an upper side open simulating the reactor pool. The cylindrical vessel is $2.9 \mathrm{~m}$ height with an inner diameter of $0.8 \mathrm{~m}$. It includes 4 transparent viewfinders to allow the flow visualization. The internals of the vessel are formed by a natural circulation tube, a lower plenum, a heating box with 24 electrical resistances representing the reactor core, and a chimney located at exit of the heating box. Surrounding the heating box there is a cylindrical chamber representing the reflector. The working fluid is distilled water. The instrumentation to be installed will provide parameter measurements such as temperature, velocity, and power. 
Figure 1: Experimental setup: vessel (1), viewfinder (2), NC tube (3), plenum (4), electrical resistances (5), chimney (6), and reflector (7).
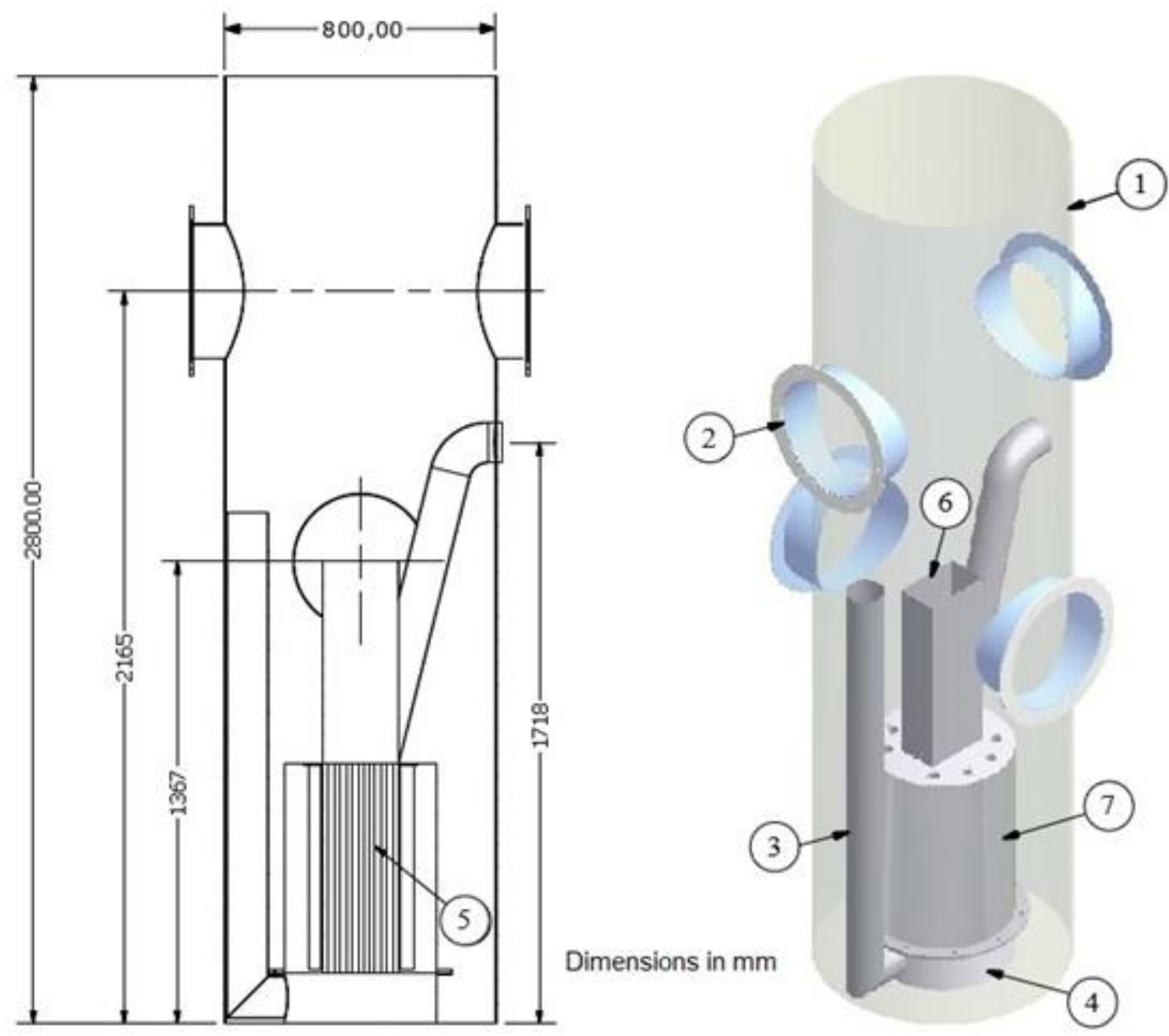

The numerical simulation of the experimental setup was carried out under single-phase natural circulation condition. The package Open FOAM was used with the $\kappa-\epsilon$ turbulence model. Besides, in the presence of the natural circulation, a laminar flow has been assumed and the NavierStokes equations of mass, momentum and energy were solved in a domain discretized by the finite volume method. The following solvers of the Open FOAM were used: "BoussinesqSimpleFoam" for the buoyancy-driven term, the "Simple" algorithm for the pressure-velocity coupling, and "Gauss Upwind" numerical scheme for the solution of the mathematical model. The unstructured mesh is formed by 900,438 elements. The initial velocity of the fluid was considered zero. The 
temperature was initialized with the value of $27^{\circ} \mathrm{C}$ for the fluid and for the entire inner part of the cylindrical vessel. For the vessel open upper surface, it is assumed room temperature, while the rest of the outer part of the vessel is regarded as being thermally insulated. Then, the heat transfer to the room was allowed on the vessel upper side only. The thermal power dissipated by the electrical resistances is $8,000 \mathrm{~W}$.

\section{RESULTS}

In this section we present results by comparing reconstructions of numerical data that are obtained by DMD and SPDMD methodologies. Firstly, we consider the traditional DMD and apply the methodology of section 3 to find the number $N$ of snapshots used to compute the dynamic modes. Next, we apply the SPDMD using the obtained $N$ to complete the analysis. The implementation is performed using Matlab resources.

The whole frame sequence has 16,077 numerical snapshots holding the velocity and temperature fields represented using a computational grid with 900,438 elements. Despite of the large number of numerical frames, we take only the last 41 ones for the analysis because they are more representative of the prototype operating temperature. In each time step, the temperature is represented as an array with 900,438 elements while the velocity field is reshaped in a vector with $3 \times 900,438=2,701,314$ entries. We start with the velocity dataset. Following the steps of section 3 , we compute the cross-correlation through expression (5), shown in Figure 2.(a). Then, the total variation technique [20] is used to process that result, to obtain a smoother signal shown in Figure 2.(b). Following, the differentiation operator is applied, given the result pictured in Figure 2.(c), that is thresholded to generate the result of Figure 2.(d) highlighting the fundamental intervals: [1, 6], $[1,35],[1,40]$. In these operations, we use the following parameters values obtained by trial and error: (1) Total variation parameters: $\lambda=0.3$ and number of iterations $N_{i t}=3$; (2) Threshold for differentiation operator result: $T=1.2 \times 10^{-9}$. 
Figure 2:(a) Cross-correlations $C_{i, i+1}$ • (b) Total variation filtering signal $\left(T(C)_{i, i+1}\right)$ obtained with $\lambda=0.3$ and $N_{i t}=3.0$. (c) Visualization of the differentiation operation. $(d)$ Intervals obtained by thresholding with $T=1.2 \times 10^{-9}$ the differentiation result: $[1,6]$, $[1,35],[1,40]$

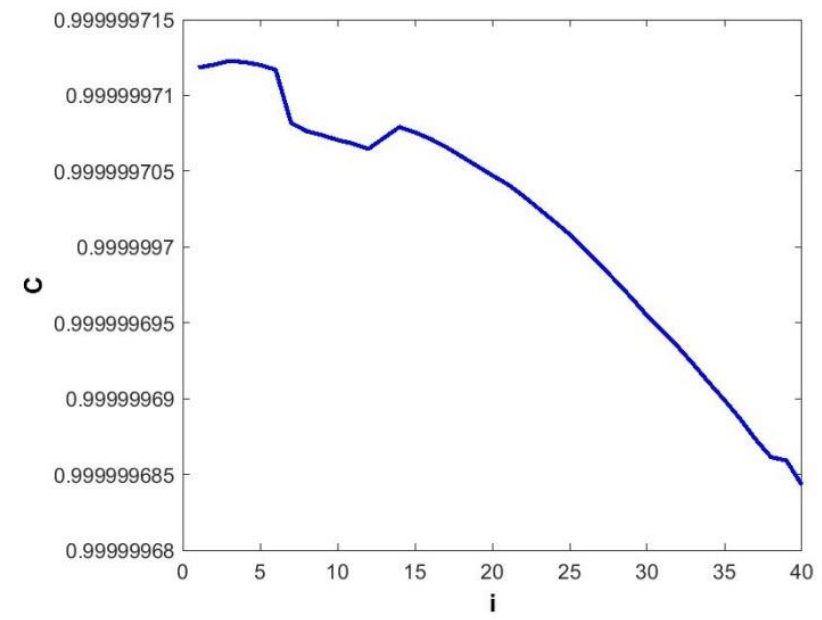

(a)

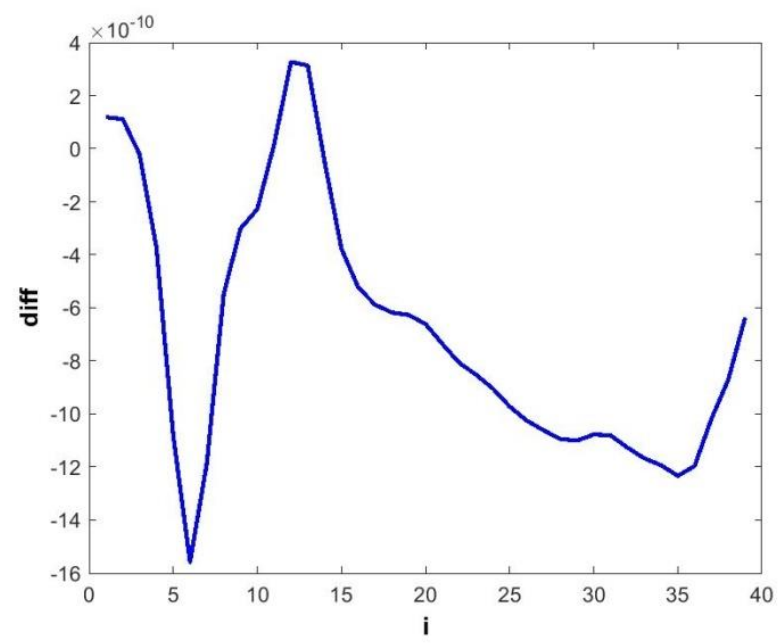

(c)

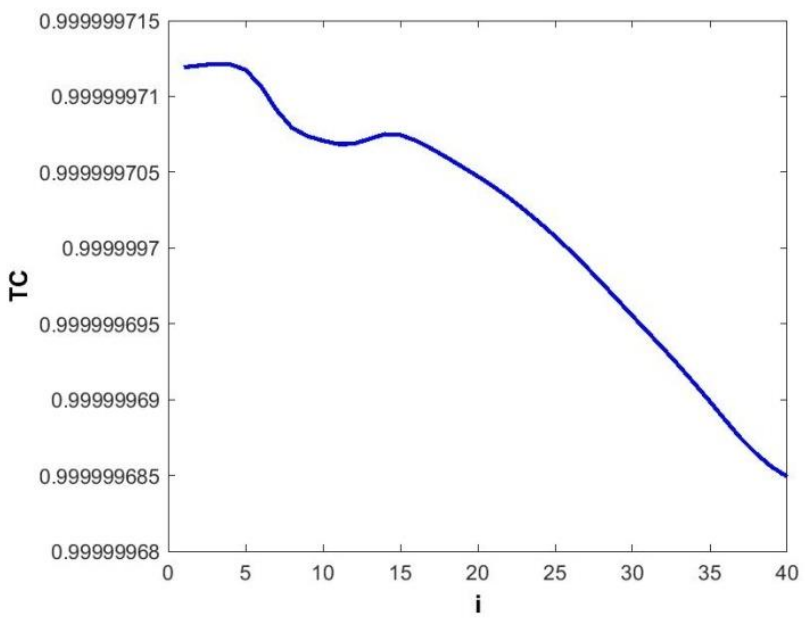

(b)

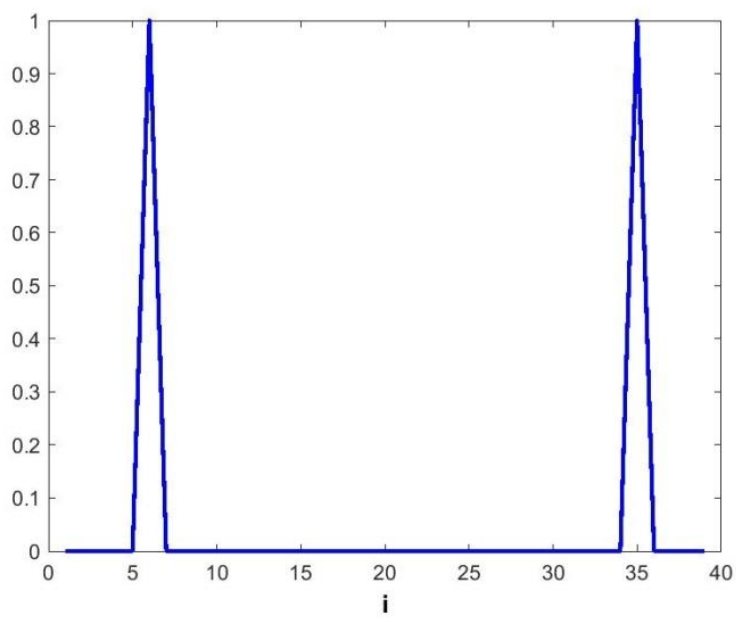

(d)

Next, we compute the traditional DMD for each interval, and perform local as well as global reconstructions using each DMD basis. In this case, the singular values of the matrices $V_{1}^{i_{j}-1}$, $\left(i_{1}=6, i_{2}=35, i_{3}=40\right)$ are non-null. So, we consider full rank matrices $\left(r=i_{j}-1\right)$ compute the DMD for each interval as well as the local reconstruction errors, given by expression (7) with 
$(k, \theta) \in\{(6,6),(35,35),(40,40)\}$,for each sub-sequence. The results are presented in Figure 3.( a).

Figure 3: (a) Local errors, computed by expression (7) with $(k, \theta) \in\{(6,6),(35,35),(40,40)\} .(b)$ Analogous result but considering the global reconstruction errors given by $R M S E_{d m d}(k, 41)$ for the intervals $[1, k]$, with $k \in\{6,35,40\}$.

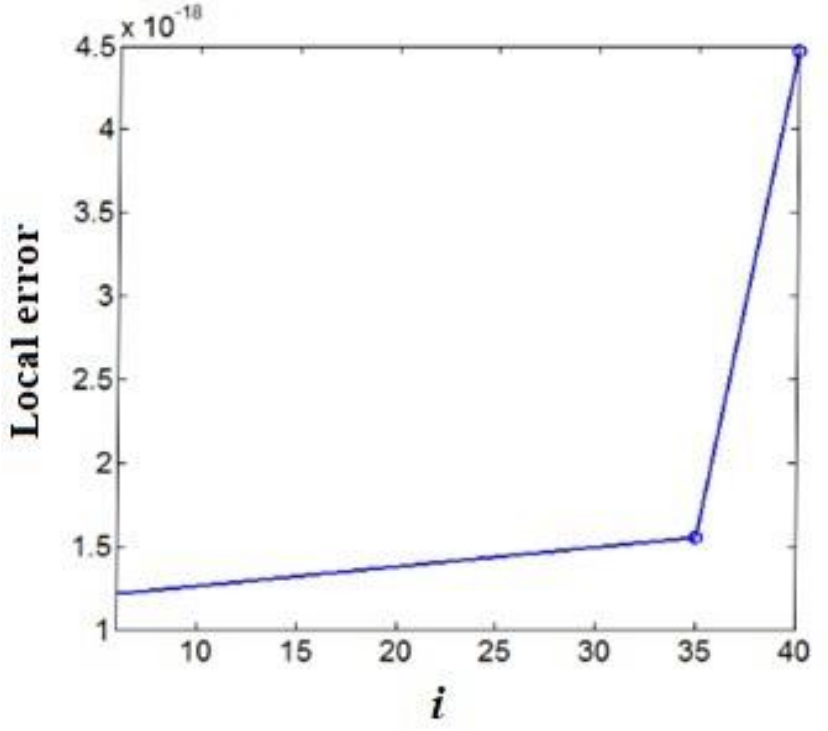

(a)

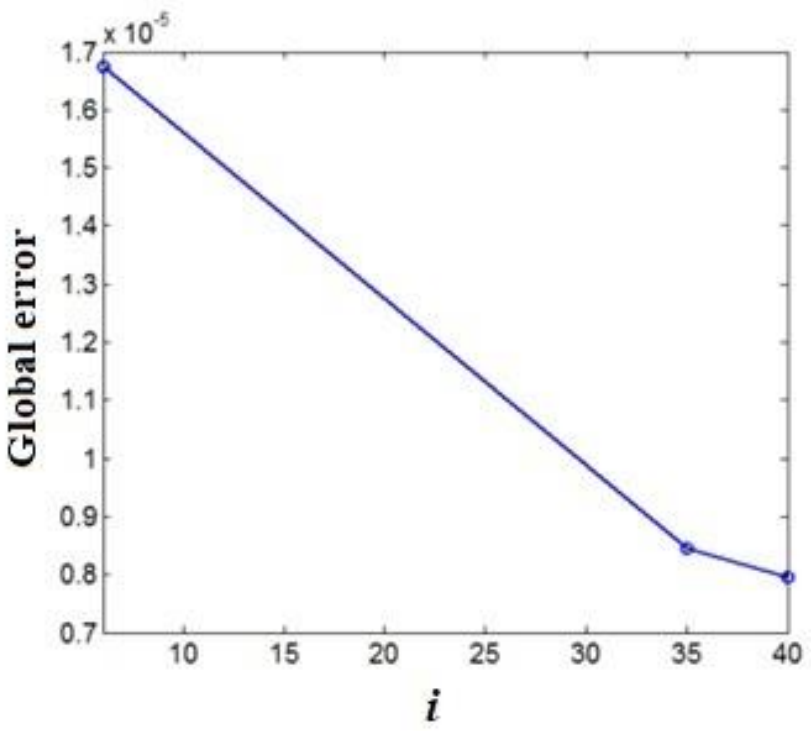

(b)

Now, we apply the SPDMD methodology using the velocity fields in the best interval $[1,40]$ to compute the dynamic modes and the sparse vector $\hat{\alpha}$ calculated following the last paragraph of section 2. In order to seek for a suitable value for parameter $\gamma$ we choose a log-distributed list compose by 20 elements logarithmically spaced from 10 to 70 . These bounds were obtained after several choices of $\gamma$ intervals with the aim to insert reasonable sparsity but keeping low global reconstruction errors. So, for each $\gamma$ value in the list we compute the SPDMD. The Figure 4.(a) shows the global reconstruction error versus the values of $\gamma$ used. We shall notice that in the interval $25.136 \leq \gamma \leq 70$ the reconstruction error and the number of non-zero alphas (Figure 4.(b)) are the minimum ones. So, we set $\gamma=25.136$ to proceed our analysis. We shall emphasize that we consider null the alpha values smaller than $10^{-12}$. 
Figure 4: (a) Global error versus gamma values for velocity fields. (b) Number of non-null alphas versus gamma values.

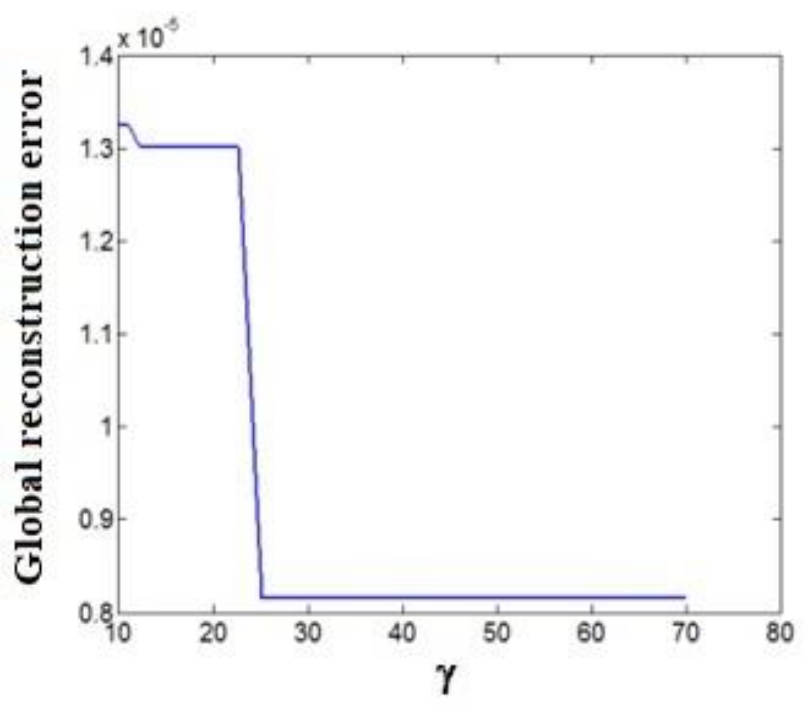

(a)

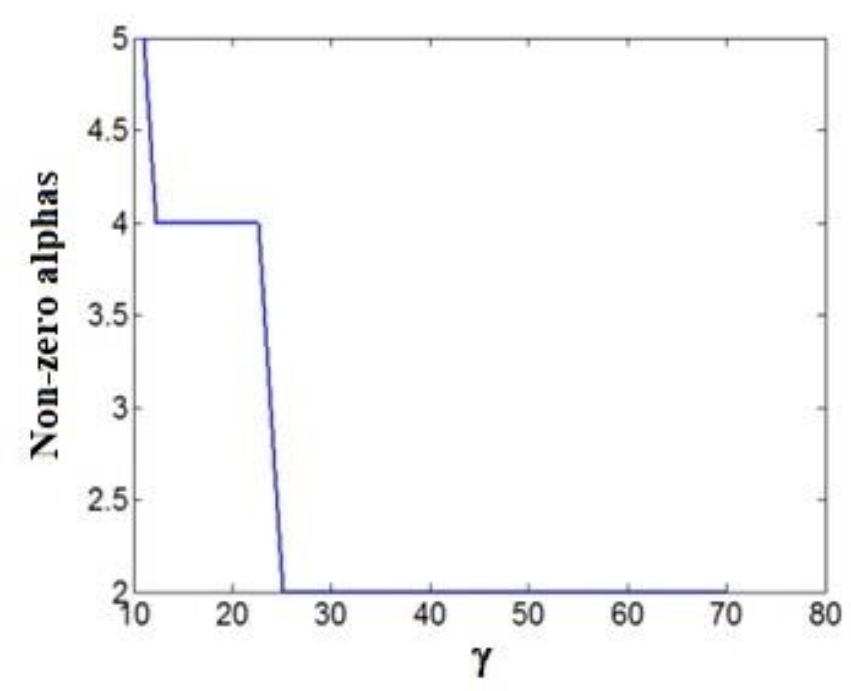

(b)

As we can see in Figure 5.(a) by observing the velocity vectors in the simulation time 16,077 seconds, the cooling water is moving along the pool (cylindrical vessel), going from the heater (core) to the top of the pool and returning back to the heater through the natural circulation tube. In the heater the water is heated (due to the heat generated by the electric resistors) and rises through the chimney. Next it moves along an upward vertical path near the wall of the cylindrical vessel. During this movement the water transfers the heat received from the heater to the pool, and then it cools. Now the water moves along a downward vertical path near the opposite wall of the cylindrical vessel going down to the natural circulation tube until the heater, where it is heated again and the heating-cooling cycle continues. The same behaviors are observed in the reconstructed data (Figures 5.(b), 5.(c)) with approximately the same visual quality. In fact, the global reconstruction errors of SPDMD and DMD are $8.1521 \times 10^{-06}$ and $7.9797 \times 10^{-06}$, respectively. Taking into account that the velocity variation falls in the range $\left[5.1993 \times 10^{-4}\right.$, $0.2980 \mathrm{~m} / \mathrm{s}$ ] such errors can be considered very low. Considering that the errors are very close one 
to each other and that SPDMD uses a sparse alpha vector (2 non-null entries among 39 ones) we can say that SPDMD outperforms DMD in the velocity field analysis.

Figure 5:(a) Visualization of the velocity field at simulation time 16, 077 seconds. (b) Same numerical frame reconstruction using DMD. (c) Reconstruction using SPDMD dynamic modes with $\gamma=25.136$.

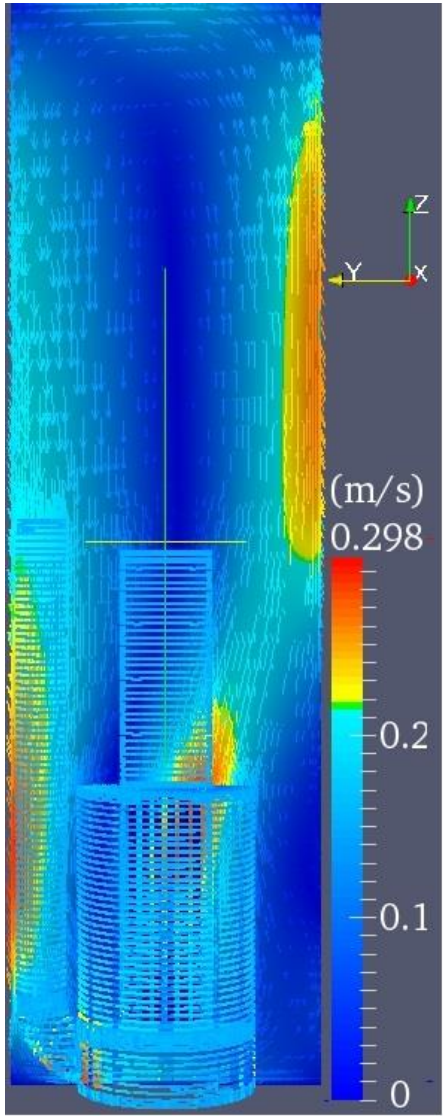

(a)

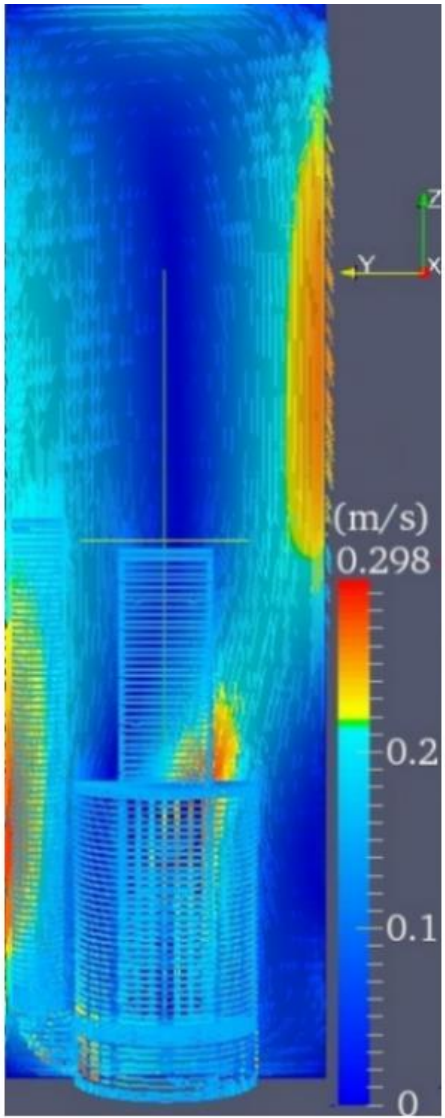

(b)

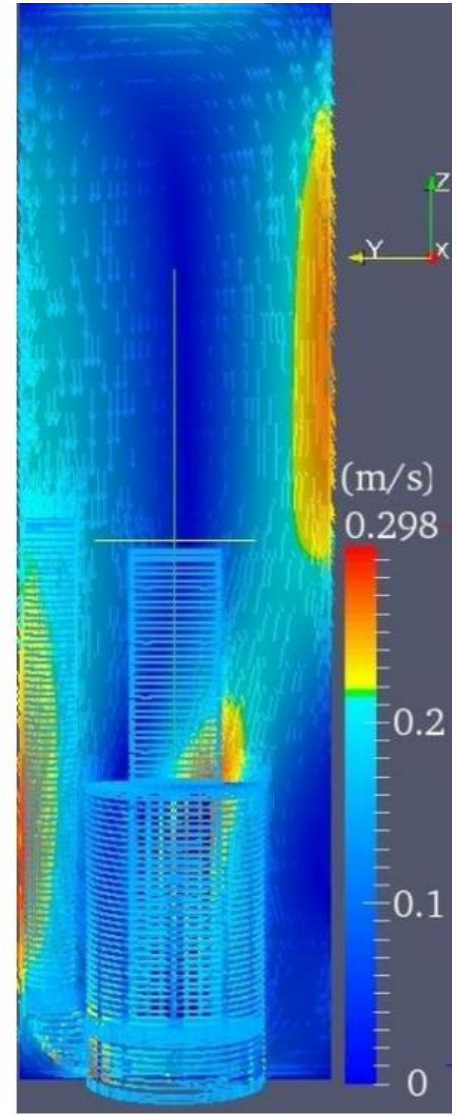

(c)

If we apply the same methodology used above to analyze the temperature field, that falls in the range [360.20,528.10] (in Kelvin), we get the intervals [1,3] and [1,40] calculated using the crosscorrelation, followed by thresholding of the differentiation. The best segment is $[1,40]$, computed by solving expression (8). Then, we seek the optimum gamma to compute SPDMD dynamic modes using a list of $20 \gamma$ values logarithmically spaced from 10 to 600. The analysis of the global reconstruction error and number of non-zero alphas versus gamma gives $\gamma=164.675$ as the best choice that generates an alpha vector with seven non-null elements. Figure 6 shows the real (Figure 
6.(a)) and reconstructed temperature fields (Figures 6.(b), 6.(c)) in the simulation time 16,077 seconds. A visual comparison between these images does not show differences between the original and reconstructed data. In fact, the global reconstruction error in this case is 0.0643 for SPDMD and 0.0680 for DMD, which are very low values in comparison with the temperature range cited above. However, once SPDMD uses a sparse alpha vector (7 non-null entries among 39 ones) and gets a global reconstruction error lower than DMD, we can say that the former outperforms the latter for the temperature data analysis.

Figure 6: (a) Temperature field at simulation time 16, 077 seconds. (b) Reconstruction using DMD. (c) Reconstruction using SPDMD dynamic modes with $\gamma$ $=164.675$.

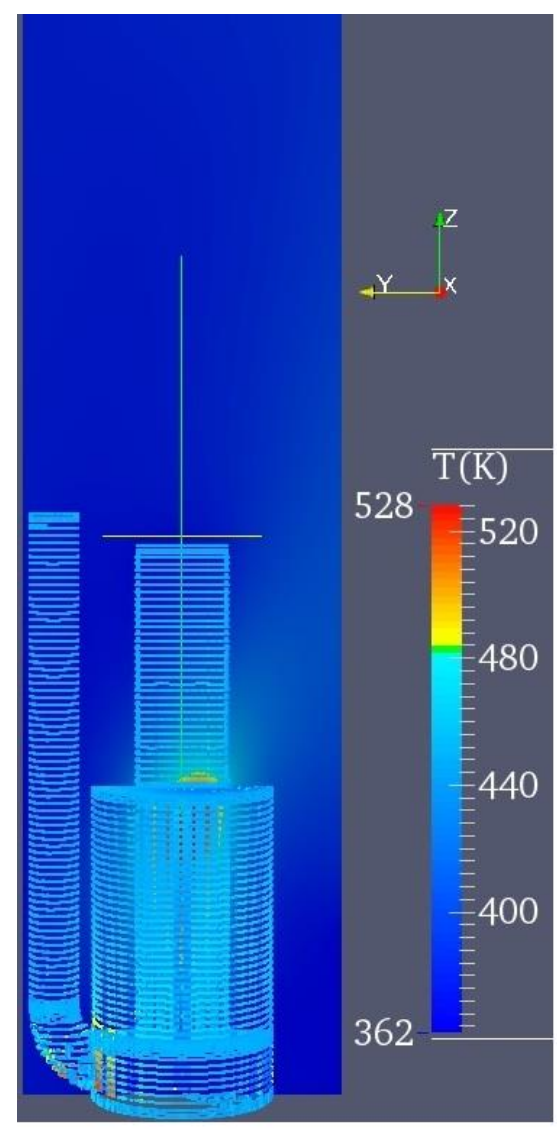

(a)

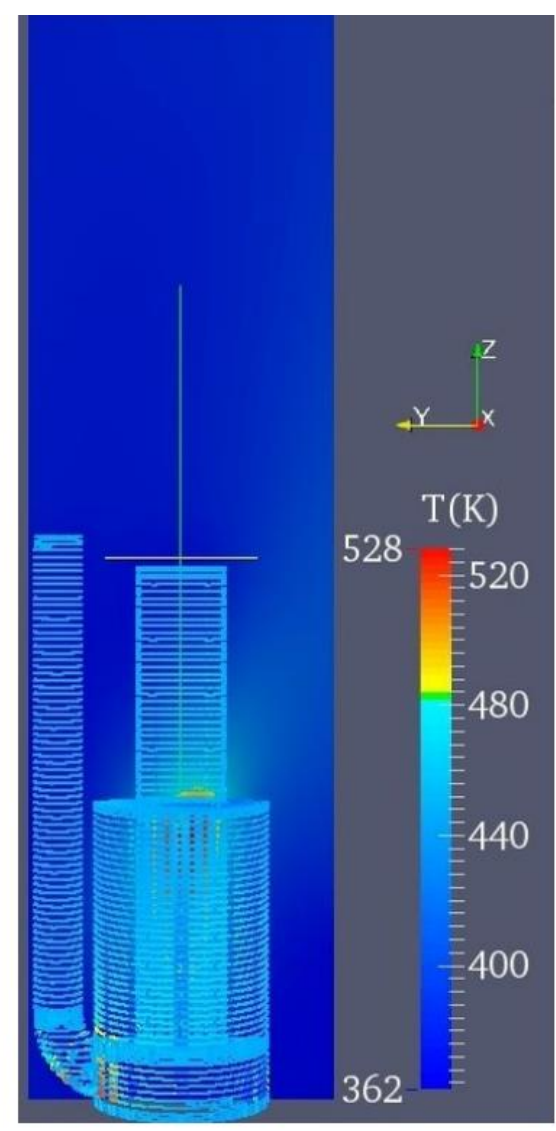

(b)

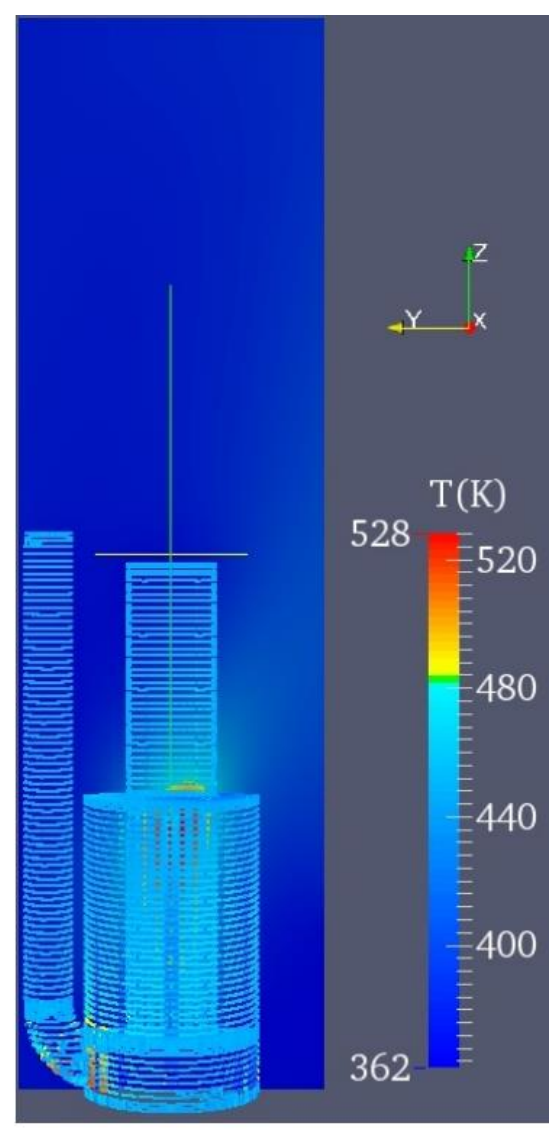

(c) 


\section{CONCLUSION}

In this work, we compare the DMD and SPDMD to analyze numerical data in natural circulation. We apply a methodology proposed in [19] to set the number $N$ of snapshots used to compute the dynamic modes. Finally, we compare DMD and SPDMD results regarding to flow preservation and compact representation. Although both SPDMD and DMD perform almost equal in terms of reconstruction quality, we notice that SPDMD outperforms DMD because it gives a more compact representation in the tests. Further works are undertaken to exploit low-rank tensor decompositions within the DMD methodology to analyze the velocity field.

\section{ACKNOWLEDGMENTS}

We thanks CAPES, CNPq and FINEP for the financial support.

\section{REFERENCES}

[1] ISHIDA, T; YORITSUNE, T. Effects of ship motions on natural circulation of deep sea research reactor DRX. Nuclear Engineering and Design, 215, p. 51-67, 2002.

[2] AZZOUNE, M.; MAMMOU, L; BOULHEOUCHAT, M. H; ZIDI, T; MOKEDDEM, M. Y; BELAID, S; BOUSBIA SALAH, A; MEFTAH, B; BOUMEDIEN, A. NUR research reactor safety analysis study for long time natural convection (NC) operation mode. Nuclear Engineering and Design, 240, p. 823-831, 2010.

[3] LU, D; XIAO, Z; CHEN, B. A new method to derive one set of scaling criteria for reactor natural circulation at single and two-phase conditions. Nuclear Engineering and Design, 240, p. 3851-3861, 2010.

[4] ZHANG, J; SHEN, X; FUJIHARA, Y; SANO, T; YAMAMOTO, T; NAKAJIMA, K. Experimental study on the safety of Kyoto University Research Reactor at natural circulation cooling mode. Annals of Nuclear Energy, 76, p. 410-420, 2015. 
[5] SHIEH, A. S; RAMSON, V. H; KRISHNAMURTY, R. RELAP5/MOD3 Code Manual, v. 6: Validation of Numerical Techniques, NUREG/CR-5535, EGG-2596, US-NRC, Washington DC, USA, 1994.

[6] VIJAYAN, P. K; AUSTREGESILO, H; TESCHENDORFF, V. Simulation of the unstable oscillatory behavior of single-phase natural circulation with repetitive flow reversals in a rectangular loop using the computer code ATHLET. Nuclear Engineering and Design, 155, p. 623-641, 1995.

[7] ANSTO. Replacement research facility - SAR chapter 1, INVAP, p. 1-58, 2004.

[8] LEE, K. Y.; YOON, H. G.; PARK, D. K. CFD analysis of a decay tank and a siphon breaker for an innovative integrated passive safety system for a research reactor. Science and Technology of Nuclear Installations, p. 1-9, 2017.

[9] ARDANEH, K.; ZAFERANLOUEI, S. A lumped parameter core dynamics model for MTR type research reactors under natural convection regime. Annals of Nuclear Energy, 56, p. 243-250, 2013.

[10]HAINOUN, A.; GHAZI, N.; ABDUL-MOAIZ, B.; MANSOUR, B. Safety analysis of the IAEA reference research reactor during loss of flow accident using the code MERSAT. Nuclear Engineering and Design, 240(5), p. 1132-1138, 2010.

[11]SHI, S.; HIBIKI, T.; ISHII, M. Startup instability in natural circulation driven nuclear reactors. Progress in Nuclear Energy, 90, p. 140-150, 2016.

[12]KARAMI, I.; AGHAIE, M. Sensitivity analysis of numerical schemes in natural cooling flows for low power research reactors. Advances in Energy Research, 5 (3), p. 255-275, 2017.

[13] SCHMID, P. J. Dynamic mode decomposition of numerical and experimental data, Journal of Fluid Mechanics, 656, p. 5-28, 2010.

[14]GOLUB, G. H.; VAN LOAN, C. F. Matrix computations, The Johns Hopkins University Press, Baltimore, USA, 1996.

[15] JOVANOVIC, M. R.; SCHMID, P. J.; NICHOLS, J. W. Sparsity-promoting dynamic mode decomposition. Phys. Fluids, 26 (2), 2014.

[16]RYSAK, A.; LITAK, G.; MOSDORF, R.; GÓRSKI, G. Investigation of two-phase flow patterns by analysis of eulerian space-time correlations. International Journal of Multiphase Flow, 85 (C), p. 23-37, 2016. 
[17] VIJAYAN, P. K.; NAYAK, A. K. Introduction to instabilities in natural circulation systems. Joint ICTP-IAEA Course on Natural Circulation Phenomena and Passive Safety Systems in Advanced Water Cooled Reactors - Lecture Notes for T-06, p. 1-30, Trieste, Italy, 2010.

[18]JOVANOVIĆ, M. R.; SCHMID, P. J.; NICHOLS, J. W. Sparsity-promoting dynamic mode decomposition code. http://people.ece.umn.edu/users/mihailo/software/dmdsp/, 2013.

[19]RAMOS, E. M.; GIRALDI, G. A.; DARZE, G. M.; FACCINI, J. L. H. Dynamic mode decomposition for analyzing two-phase flows video data, In: 10th INTERNATIONAL CONFERENCE ON MULTIPHASE FLOW (ICMF), Rio de Janeiro, Brazil, May 19-24, p. $1-10,2019$.

[20]SELESNICK, I. W.; PAREKH, A.; BAYRAM, I. Convex 1-d total variation denoising with non-convex regularization. IEEE Signal Processing Letters, 22, p.141-144, 2015. 